\title{
Crosslinking of epoxy-polysiloxane system by reactive blending
}

\author{
M. Gonzalez ${ }^{\text {b }}$ P. Kadlec ${ }^{\mathrm{a}}$, P. Štěpánek ${ }^{\mathrm{a}}$, A. Strachota ${ }^{\mathrm{a}}$, L. Matějka ${ }^{\mathrm{a}, *}$ \\ anstitute of Macromolecular Chemistry, Academy of Sciences of the Czech Republic, Heyrovský Sq. 2, 16206 Prague 6, Czech Republic \\ ${ }^{\mathrm{b}}$ Technological Institute Álvaro Alonso Barba, University Carlos III Madrid, Av. Universidad 30, 28911 Leganés, Madrid, Spain
}

\begin{abstract}
The epoxy-polysiloxane network was prepared by reactive blending of poly[(3-aminopropyl)methylsiloxane] (PAMS) containing pendant amino groups and diglycidyl ether of Bisphenol A (DGEBA). The initially immiscible blend is compatibilized during the reaction and crosslinked. Network formation, dynamics of the system and evolution of morphology were determined by dynamic mechanical analysis and light scattering techniques. The grafting epoxy-amine reaction involves a high extent of cyclization resulting in a high fraction of the sol in the networks. Dynamic light scattering data analysis reveals fast and slow relaxation modes of reacting species in the pregel and one single mode in the post-gel state. The network with a stoichiometric composition shows the most homogeneous morphology with a single glass transition temperature. On the contrary, the networks with excess of PAMS are strongly phase-separated exhibiting the unreacted PAMS-rich phase, PAMS phase partly grafted with epoxide and PAMS-DGEBA crosslinked phase.
\end{abstract}

Keywords: Reactive blending; Siloxane network;

\section{Introduction}

Modification of brittle epoxy systems with the aim to increase impact strength and fracture toughness is usually performed with elastomers or thermoplastics. Polysiloxanes are proper toughening agents due to their low glass transition temperature, good thermal and oxidative stability, low surface tension as well as high flexibility and good weather ability. However, epoxy and polysiloxane systems are immiscible and their compounding is difficult. Recently, these incompatible materials were processed by reactive blending resulting in compatibilization during the reaction. Cabanelas et al. [1] has studied morphology of the epoxypolyorganosiloxane network prepared from diglycidyl ether of Bisphenol A (DGEBA) and a polysiloxane functionalized with reactive pendant amino groups, poly[(3-aminopropyl)methylsiloxane) (PAMS). The blend PAMS-DGEBA is initially immiscible at room temperature; however, grafting of DGEBA to the polysiloxane chain by the epoxy-amine reaction leads to partial compatibilization and crosslinking of the blend. Nevertheless, the cured networks are

\footnotetext{
* Corresponding author. Tel.: +420-296-809-281; fax: +420-296-809410.

E-mail address: matejka@imc.cas.cz (L. Matějka).
}

heterogeneous as proved by transmission microscopy and microfluorescence spectroscopy [1] with PAMS-rich domains irregularly dispersed in the epoxy-rich matrix. The networks with stoichiometric composition exhibit the co-continuous structure with the relatively homogeneous morphology compared with systems containing excess of epoxy or amine. The size of PAMS-rich domains increases with PAMS content; optical microscopy shows a structure gradient in the dispersed phase due to diffusion restriction of DGEBA in the PAMS phase.

In this paper, we have followed crosslinking of the reactive blend PAMS-DGEBA and formation of the epoxysiloxane network by using chemorheology and light scattering technique. Reactive blending generally is based on competition of several factors. These include the rate of the compatibilization reaction, thermodynamics of the phase state in dependence on the structure changes and gelation of the system arresting the existing morphology. Therefore, in order to be able to explain and tune the final PAMS-DGEBA morphology, the reaction kinetics and polymer structure evolution during crosslinking including gelation and phase state development were studied. Final morphology, network structure and thermomechanical properties of the cured PAMS-DGEBA systems as a 
function of composition were determined using dynamic mechanical analysis (DMA).

\section{Experimental}

\subsection{Synthesis and crosslinking of poly[(3-aminopropyl) methylsiloxane]}

Poly[(3-aminopropyl) methylsiloxane] (PAMS) was prepared by sol-gel polymerization of (3-aminopropyl)methyldiethoxysilane (AMDES). The reaction consisting in hydrolysis and condensation of the monomer was carried out under nitrogen at $60{ }^{\circ} \mathrm{C}$ for $2 \mathrm{~h}$ with $100 \%$ water excess over stoichiometric composition. The product was characterized by SEC (mixed-E column, Polymer Laboratories) using polystyrene standards. The possible adsorption processes in column were not significant to affect interpretation of SEC data. All products were eluted before unretained solute (toluene) indicating absence of adsorption. SEC peaks were symmetric, no tailing was observed. The molecular weights are: $M_{\mathrm{n}}=500, M_{\mathrm{w}}=630$. The polymerization degree; $P_{\mathrm{n}} \approx 4.5, P_{\mathrm{w}} \approx 5.5$ equals to the number of pendant $\mathrm{NH}_{2}$ groups and corresponds to the number and weight-average functionality of reactive $\mathrm{NH}$ groups in PAMS, $f_{\mathrm{n}} \approx 9, f_{\mathrm{w}} \approx 11$. The determined molecular weights, however, are low compared to literature data [2]. Therefore, also an effect of a higher average functionality of PAMS is taken into account in order to avoid the wrong conclusions because of a possible analysis error.

PAMS was crosslinked by the reaction with diglycidyl ether of Bisphenol A (DGEBA) either in a heterogeneous blend or in the system homogenized with 10 or $20 \%$ THF. Kinetics and structure evolution were studied at 40 or $50{ }^{\circ} \mathrm{C}$. For characterization of crosslinked products and study of mechanical properties, the networks were prepared by curing at $120{ }^{\circ} \mathrm{C}$ and vacuum-dried. Composition of the reactive system was characterized by mole ratio of functional $\mathrm{NH}$ groups of PAMS and epoxy groups in DGEBA, $r_{\mathrm{AE}}=\mathrm{NH} /$ epoxy.

AMDES (Fluka) was used as received and DGEBA was purified by crystallization.

\subsection{Methods}

\subsubsection{IR spectroscopy}

Kinetics of the reaction of the amine and epoxy reagent was followed by IR spectroscopy using FTIR Perkin Elmer Paragon 1000 PC spectrometer. The peak at $914 \mathrm{~cm}^{-1}$ corresponding to the epoxy group was used to determine the reaction conversion. The reactions of the model systems; phenyl glycidyl ether (PGE)-AMDES and PGE-PAMS; were performed in vials and for IR measurements the reagents were dissolved in chloroform. The reaction mixture
DGEBA-PAMS homogenized with $10 \%$ THF was measured as a thin film on $\mathrm{KBr}$ pellets.

\subsubsection{Dynamic mechanical analysis}

DMA was carried out with an ARES apparatus (Rheometric Scientific). Evolution of dynamic viscosity and complex shear modulus during polymerization was followed in parallel-plate geometry using oscillatory shear deformation at a frequency of $1 \mathrm{~Hz}$. The multiwave frequency mode was used for the gel point evaluation. Rectangular cured network samples of the size $5 \times 1 \times 0.2 \mathrm{~cm}$ were subjected to measurement of complex shear modulus as a function of temperature from -120 up to $160{ }^{\circ} \mathrm{C}$ at a shear deformation frequency of $1 \mathrm{~Hz}$.

\subsubsection{Light scattering}

During polymerization, we have measured the time dependence of the intensity of light scattered at angle $90^{\circ}$ as well as the dynamic light scattering (DLS) from the sample. The samples were filtered in order to remove dust and other particles which could interfere in the measurements.

The DLS instrument employed an ALV5000/E correlator. The integral intensity of the light passing through the sample was measured by photodiode and the integral intensity of the light scattered at angle $90^{\circ}$ was recorded as a part of the DLS measurement. The light source was a Hewlett-Packard 125A laser with wavelength $\lambda_{0}=632 \times$ $\mathrm{nm}$. The scattered light was fed into an avalanchephotodiode double detector via a multimode optical fiber. In this unit, the signal is split by a beam splitter into the two photodetectors and the correlator is operated in the crosscorrelation mode, which ensures that all artifacts due to afterpulsing and other imperfections do not affect the correlation function in the early time regime. The measured intensity correlation functions $g^{2}(t)$ were analyzed using non-linear inverse Laplace transformation to obtain the distributions of relaxation times $B(\tau)$ according to Eq. (1), where $\beta$ is an instrumental parameter.

$g^{2}(t)-1=\left[\beta \int B(\tau) \exp (-t / \tau) \mathrm{d}(\tau)\right]^{2}$

The REPES [3] transformation program was used. This program differs from the widely used program CONTIN in that it fits directly the measured intensity correlation function $g^{2}(t)$.

\section{Results and discussion}

The incompatible blend PAMS-DGEBA forms an epoxy-siloxane network by the epoxy-amine crosslinking reaction of the diepoxide with pendant amine functionalities of PAMS. We have studied kinetics of the epoxy-amine reaction, evolution of the polymer structure including gelation as well as phase state evolution during crosslinking. IR spectroscopy, chemorheology and light scattering 
measurements were used to follow the reaction. In addition to study of the blend bulk also measurements were carried out using a small amount of THF to partly homogenize the system. The cured networks were characterized by DMA and by the sol-gel analysis. Both stoichiometric and nonstoichiometric networks were prepared and the effect of composition $r_{\mathrm{AE}}$ on the final morphology and mechanical properties was determined.

\subsection{Reaction kinetics}

The reactivity of the PAMS-DGEBA blend was compared with low-functional model systems involving monomers; phenyl glycidyl ether (PGE) and AMDES. Fig. 1 shows the course of the reaction characterized by disappearance of the epoxy groups in the stoichiometric reaction mixtures AMDES-PGE, PAMS-PGE and PAMSDGEBA ( $10 \%$ of THF). The results reveal that the reactivity of the amino groups in PAMS does not differ from that in the monomer AMDES in the reaction with PGE. There are no steric restrictions affecting the reaction on the polymer chain. However, in the case of PAMS-DGEBA the reaction is slowed down at later stages at conversion of epoxy groups $\sim 50 \%$, which cannot be explained merely by the effect of dilution with THF. This is assumed to be a result of diffusion control as discussed below. While the epoxy conversion in AMDES-PGE and PAMS-PGE is complete at $T=50{ }^{\circ} \mathrm{C}$ in $2 \mathrm{~h}$, the cured PAMS-DGEBA still contains $10 \%$ of unreacted epoxy groups after 1 -day reaction. The final conversion in the bulk system cured at $120^{\circ} \mathrm{C}$ was $95 \%$.

\subsection{Evolution of the structure, phase state and gelation of the system}

\subsubsection{Chemorheology}

The structure evolution during crosslinking reaction of PAMS-DGEBA was characterized by an increase in dynamic viscosity. Fig. 2 shows viscosity evolution in the reaction of the bulk system and in the THF solution (10\% of

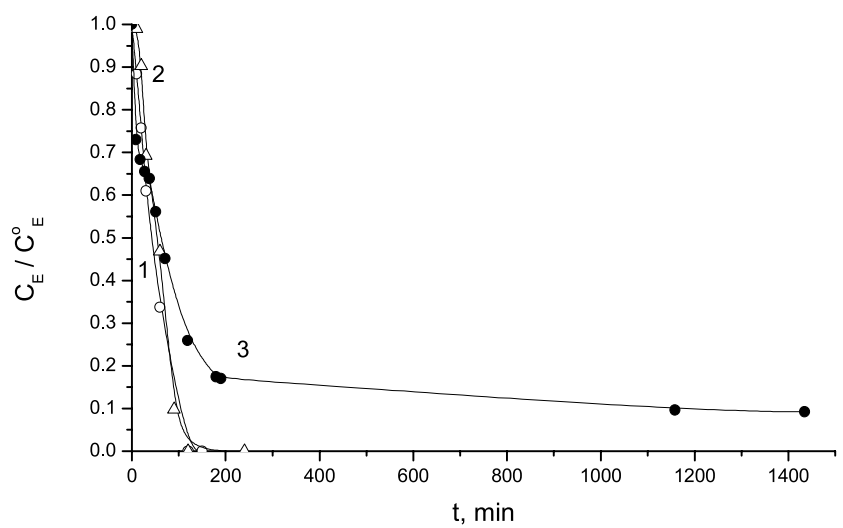

Fig. 1. Kinetics of the reaction AMDES-PGE (1 O), PAMS-PGE $(2 \triangle)$ and PAMS-DGEBA (10\% of THF) $(3-)$ at $50{ }^{\circ} \mathrm{C}$.

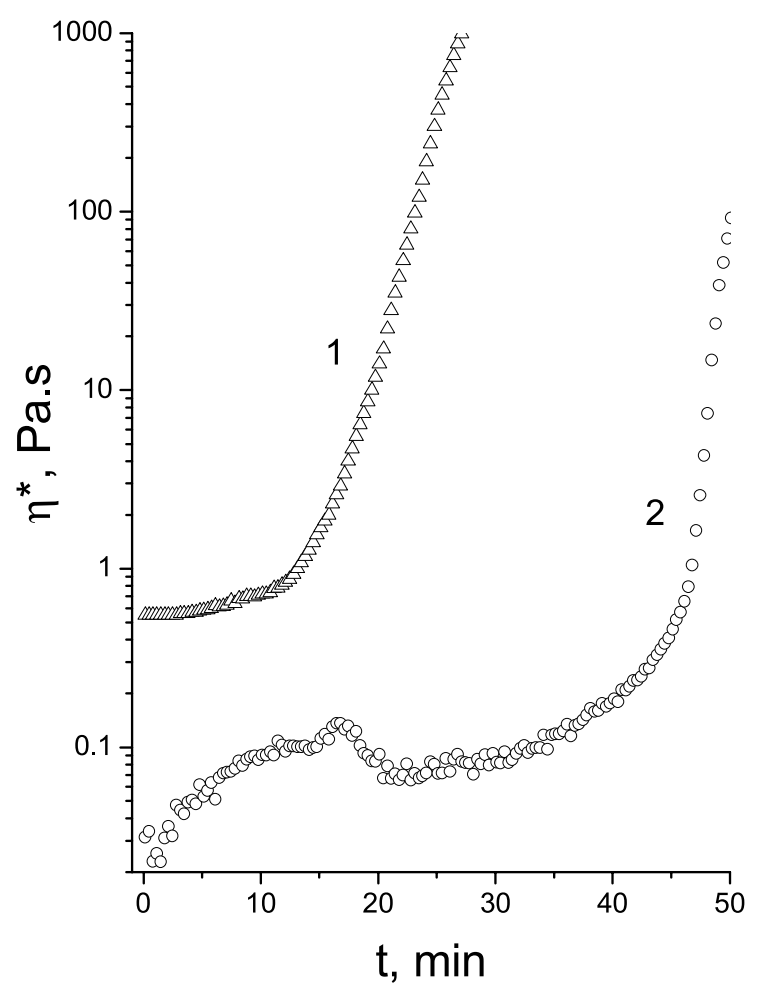

Fig. 2. Evolution of the dynamic viscosity during the reaction PAMSDGEBA at $50{ }^{\circ} \mathrm{C} .1$ bulk blend, 2 solution in THF (10\% of THF).

THF). While in the bulk, viscosity monotonically grows during the reaction, in the solution a sudden viscosity decrease in the early reaction stage occurs followed by another viscosity rise. This phenomenon is brought about by phase separation [4] as discussed below. The steep increase in viscosity indicates gelation of the system. The gel time was determined by using a multiwave frequency mode measurement and taking into account a power law behaviour at the gel point: [5] $G^{\prime}(\omega) \sim G^{\prime \prime}(\omega) \sim \omega^{n}$, where $G^{\prime}, G^{\prime \prime}$ are dynamic storage and loss shear moduli, $n$ is the critical relaxation exponent and $\omega$ is the experimental measurement frequency. At the gel point, the loss factor $\tan \delta\left(=G^{\prime \prime} / G^{\prime}\right)$ is independent of the measurement frequency. The bulk PAMS-DGEBA system of a stoichiometric composition gels in $\sim 25 \mathrm{~min}$ or in $60 \mathrm{~min}$ at $50{ }^{\circ} \mathrm{C}$ and at $40{ }^{\circ} \mathrm{C}$, respectively. Dilution of the reaction mixture with $\mathrm{THF}$ results in slowing down of the crosslinking; at $50{ }^{\circ} \mathrm{C}$ gelation occurs in $50 \mathrm{~min}$ or in $80 \mathrm{~min}$ in the presence of 10 or $20 \% \mathrm{THF}$, respectively. The value of the loss factor at the gel point was $(\tan \delta)_{\mathrm{C}}=2.2$. This value was employed to calculate the critical exponent $n=$ 0.73 using the relation, $n=2 \delta_{\mathrm{C}} / \pi$ [5].

The conversion of the epoxy groups in the stoichiometric PAMS-DGEBA (10\% of THF) system at the point of gelation is $\left(\alpha_{\mathrm{E}}\right)_{\mathrm{C}}=0.47$. The classic theory of network formation [6] (Eq. (2)) predicts the value $\left(\alpha_{\mathrm{E}}\right)_{\mathrm{C} \text {,th }}=0.32$ for an ideal random epoxy-amine reaction assuming bifunctional epoxide, $f_{\mathrm{E}}=2$, and the weight average functionality 


$$
\begin{aligned}
& \text { of PAMS, } f_{\mathrm{wA}}=11: \\
& \begin{array}{c}
\left(\alpha_{\mathrm{A}} \alpha_{\mathrm{E}}\right)_{\mathrm{C}}=\left[\left(f_{\mathrm{E}}-1\right)\left(f_{\mathrm{wA}}-1\right)\right]^{-1} \\
\left(\alpha_{\mathrm{E}}\right)_{\mathrm{C}}=\sqrt{\left(f_{\mathrm{wA}}-1\right)^{-1}} \text { for stoichiometric } \\
\text { composition }\left(\alpha_{\mathrm{A}}=\alpha_{\mathrm{E}}\right)
\end{array}
\end{aligned}
$$

where $\alpha_{\mathrm{E}}$ and $\alpha_{\mathrm{A}}$ are conversion of epoxy and $\mathrm{NH}$ groups of DGEBA and PAMS, respectively. The delay of gelation with respect to the theory could be explained by cyclization in the reaction of DGEBA with two neighboring $\mathrm{NH}_{2}$ groups in PAMS thus not contributing to the crosslinking of the system. Slowing down of the reaction compared to the models PAMS-PGE and AMDES-PGE at $\alpha_{\mathrm{E}}>0.5$ shown in Fig. 1 correlates approximately with time of gelation. Restricted chain mobility in the crosslinked system and diffusion control of the reaction could be a reason for this deceleration. This is in agreement with concentration gradients in dispersed PAMS-rich phases observed by Cabanelas et al. [1].

\subsubsection{Time resolved light scattering}

The phase state of the system during crosslinking was followed using light scattering and turbidity measurements that characterize phase separation. Because of a very high turbidity of the blend, we have studied also systems homogenized with 10 and $20 \%$ of THF. In time-resolved light scattering (TRLS) experiments, we have measured intensity of the scattered light during the reaction.

The cloudy PAMS-DGEBA blend becomes slightly more transparent at the early stage of the reaction. However, this change in transparency cannot be observed in the TRLS measurement due to too high turbidity. Fig. 3(a) shows the intensity of the scattered light $I$ during crosslinking at $40{ }^{\circ} \mathrm{C}$. While the experiment is insensitive to early changes of the scattered intensity, a significant increase in the intensity along with an enhancement in intensity fluctuations are observed in ca. 50-60 min after starting the reaction, which correlates with gelation of the blend. A different phase state evolution was observed in a transparent THF solution (10\% of THF) in the reaction at $50{ }^{\circ} \mathrm{C}$. In this case, three regions can be discerned in Fig. 3(b); (a) increase in I reaching the local maximum in $\sim 15 \mathrm{~min}$, (b) decrease in $I$ and another growth to the maximum value at $\sim 50 \mathrm{~min}$, (c) an approximately constant intensity value at $t>90 \mathrm{~min}$. A qualitatively similar TRLS result was obtained in the case of the reaction with $20 \%$ of THF (Fig. 3(c)). Several competing processes take place during the reaction and we assume that the experimental data could be interpreted as follows: In THF solution, the polymer structure growth and crosslinking result in a tendency to phase separation from THF solution while the epoxy-amine grafting reaction leads to compatibilization of the reactive blend. The increase in the scattered light intensity at the early reaction stage in the solution in Fig. 3(b) coincides with the sudden decrease in viscosity observed in Fig. 2 and corresponds to phase
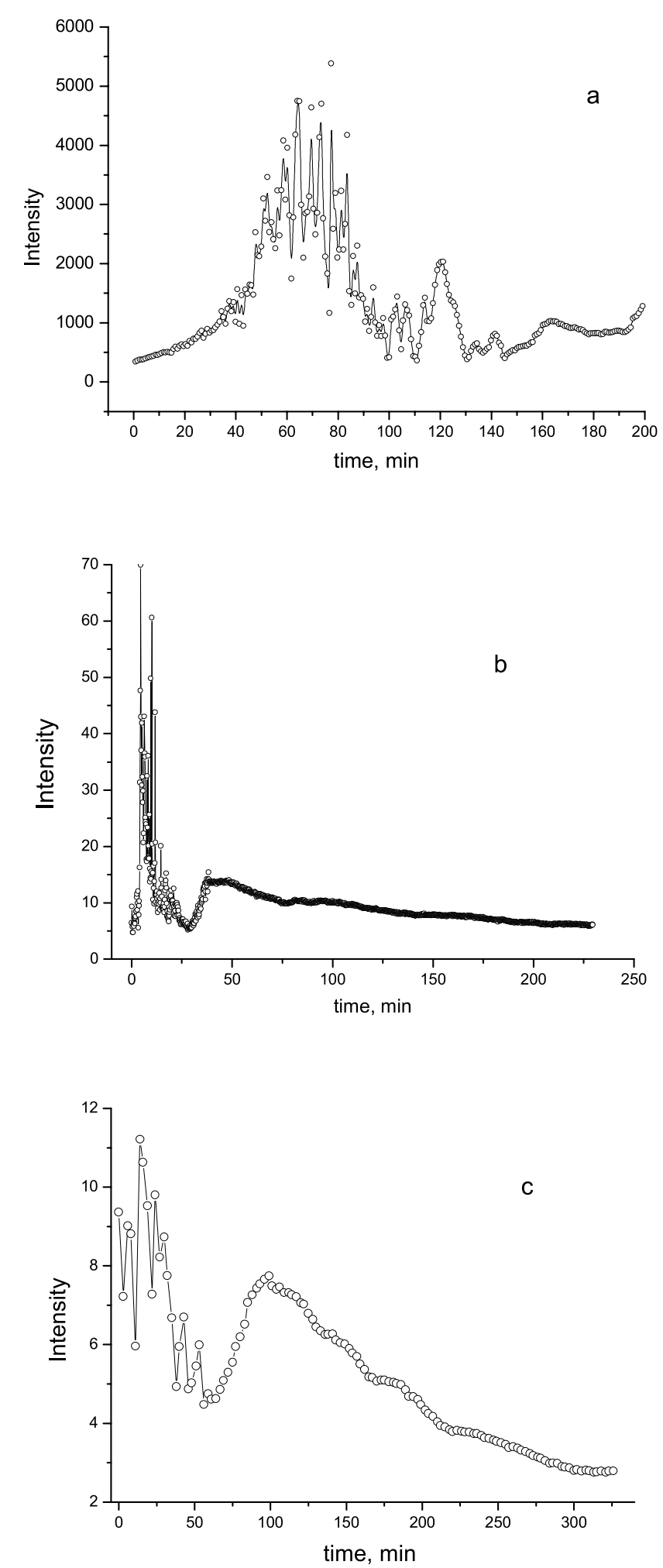

Fig. 3. Evolution of the intensity of the scattered light during the reaction PAMS-DGEBA (a) bulk, $T=40{ }^{\circ} \mathrm{C}$, (b) $10 \% \mathrm{THF}, T=50{ }^{\circ} \mathrm{C}$, (c) $20 \%$ $\mathrm{THF}, T=50^{\circ} \mathrm{C}$.

separation of a reactive blend from THF. No such viscosity and scattered intensity change was observed in the bulk system. The grafting reaction then leads to an increase in miscibility and $I$ drops. Large intensity fluctuations in the 
bulk and the enhancement of $I$ at a later reaction stage, both in the bulk and in solution, correlate with gelation of the system determined independently. The gel formation fixes the existing structure, which explains the stabilization of the intensity for longer times since after gelation of the system large-scale fluctuations in the local composition of the system are no longer possible.

\subsubsection{Dynamic light scattering}

Dynamics of the forming structure in the partially homogenized PAMS-DGEBA solutions in THF was determined by DLS. The reaction was conducted in a sealed glass cell at $50{ }^{\circ} \mathrm{C}$. The scattered light was collected from the sample during the reaction at an angle of $90^{\circ}$. Correlation functions were measured successively for $5 \mathrm{~h}$, each correlation function was accumulated for a period of $300 \mathrm{~s}$. Although the reaction proceeds during the measurement, it was found that each correlation function accumulated over a period of $300 \mathrm{~s}$ was representative of the dynamic processes occurring in the sample in that time interval. Fig. 4 shows representative correlation functions obtained before and after the gel point that occurs at a reaction time of $50 \mathrm{~min}$ for a sample containing $10 \%$ of THF. Before the gel point, two dynamic processes are clearly observable and only one after the gel point. We observe also a remarkable drop in the amplitude of the correlation function after the gel point, which is due to the beginning of non-ergodic scattering once the gel point has been passed. The amplitude of the correlation function (parameter $\beta$ in Eq. (1)) is in fact a very sensitive indication of the exact location of the gel point as demonstrated in Fig. 5. The gel time of 59 min evaluated from Fig. 5 corresponds

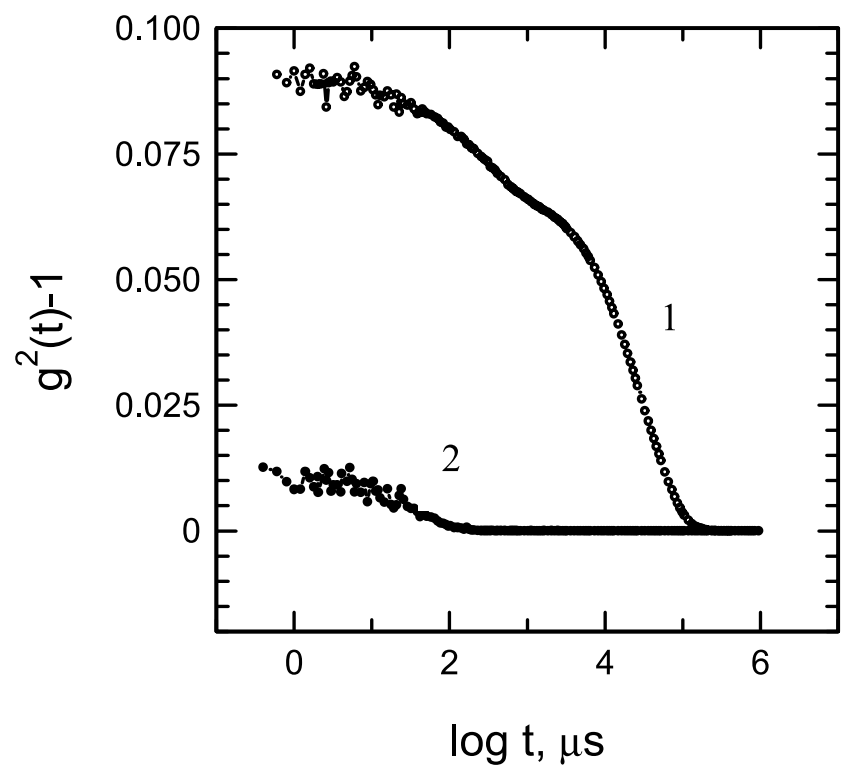

Fig. 4. Intensity correlation functions during crosslinking PAMS-DGEBA $\left(10 \%\right.$ of THF). 1 -pregel stage, $t_{\text {reaction }}=16 \mathrm{~min}, 2$-postgel stage, $t_{\text {reaction }}=4 \mathrm{~h}$. well with the gel time determined from chemorheology and TRLS.

Distributions of relaxation times obtained from the correlation functions in Fig. 4 are shown in Fig. 6. The position of these modes as a function of reaction time is displayed in Fig. 7. We assume that all three modes exhibit a diffusive behavior, $\Gamma=D q^{2}$, (with $q$ being the scattering vector, $D$ the diffusion coefficient and $\Gamma(=1 / \tau)$ the relaxation rate) although it was not possible to verify it experimentally, since measurements performed at different scattering angles would correspond to different reaction times.

Before gel time, the fast mode (' 1 ') corresponds to mutual diffusion of the unreacted components. The relaxation time of this mode increases as the reaction proceeds because the viscosity of the medium increases (see Fig. 7). The slow mode ('2') corresponds to diffusion of pregel PAMS-DGEBA clusters, which increase in size as the reaction proceeds. The relaxation time of this mode increases even faster than that of mode 1 because of the combination of both effects-increase in viscosity and cluster size. The size $R$ of the pregel cluster was calculated according to the Stokes-Einstein relation, $D=k T / 6 \pi \eta R$, using the value of the viscosity of the reaction mixture at the beginning of the reaction $\left(\eta^{*}=0.025 \mathrm{~Pa} \mathrm{~s}\right)$. The cluster size was determined to be $R \sim 100 \mathrm{~nm}$ at the beginning of the reaction. At later times in the pregel stage, we can no longer apply the Stokes-Einstein relation, which assumes diffusion of non-interacting objects of low number density in a medium of viscosity $\eta$.

After gel point, a single relaxation time (mode ' 3 ') is observed that corresponds to the dynamics of the part of the polymer chain between crosslinks. Since the dependence of the relaxation time on the reaction time is rather weak we

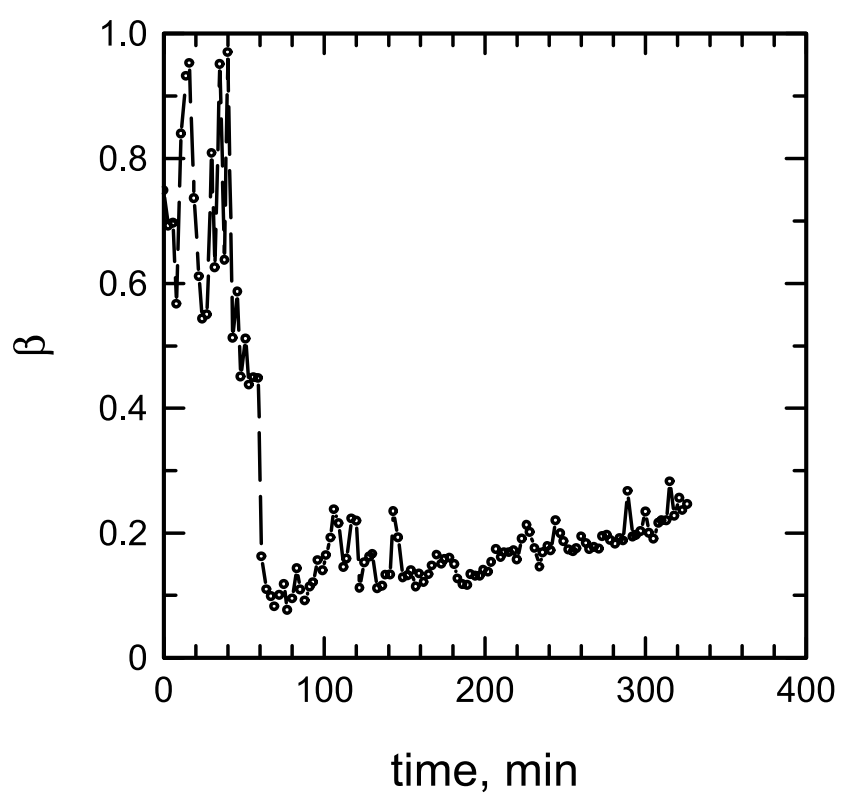

Fig. 5. Initial amplitude of correlation function $\beta$ as a function of reaction time. 

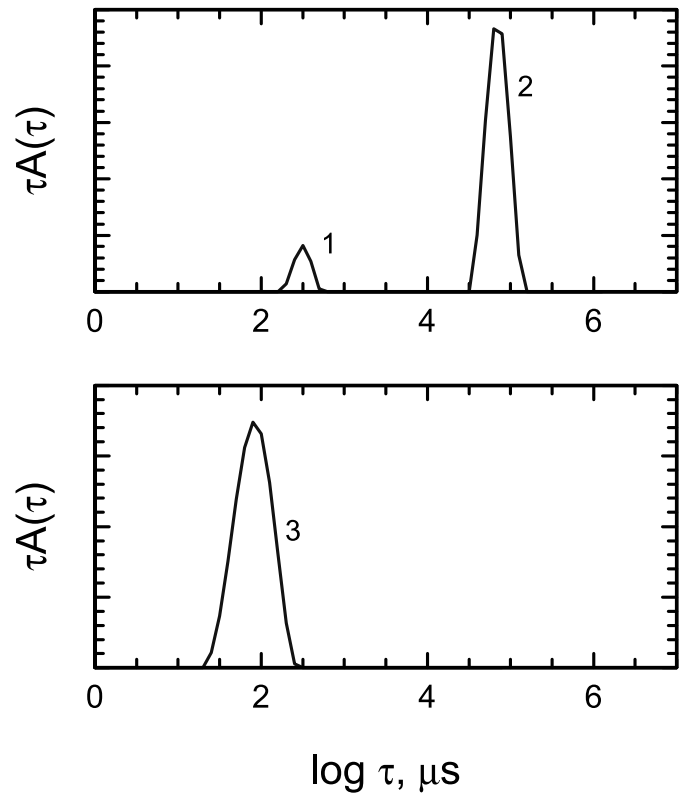

Fig. 6. Distribution of relaxation times in the reaction PAMS-DGEBA ( $10 \%$ of THF). (a) pregel stage, $t_{\text {reaction }}=16 \mathrm{~min}$, (b) postgel stage, $t_{\text {reaction }}=4 \mathrm{~h}$.

were able to establish that mode ' 3 ' has a diffusive character. Fig. 8 shows that, indeed, $\Gamma \sim q^{2}$ as required in such a case.

At the gel point and in its immediate vicinity the situation is different. As the gel point is approached, the width of the slow pregel relaxation (mode ' 2 ' in Fig. 6) continuously increases until at the gel point the relaxation corresponding to this mode is well described by a power law behavior. The correlation function of scattered light can then be modeled

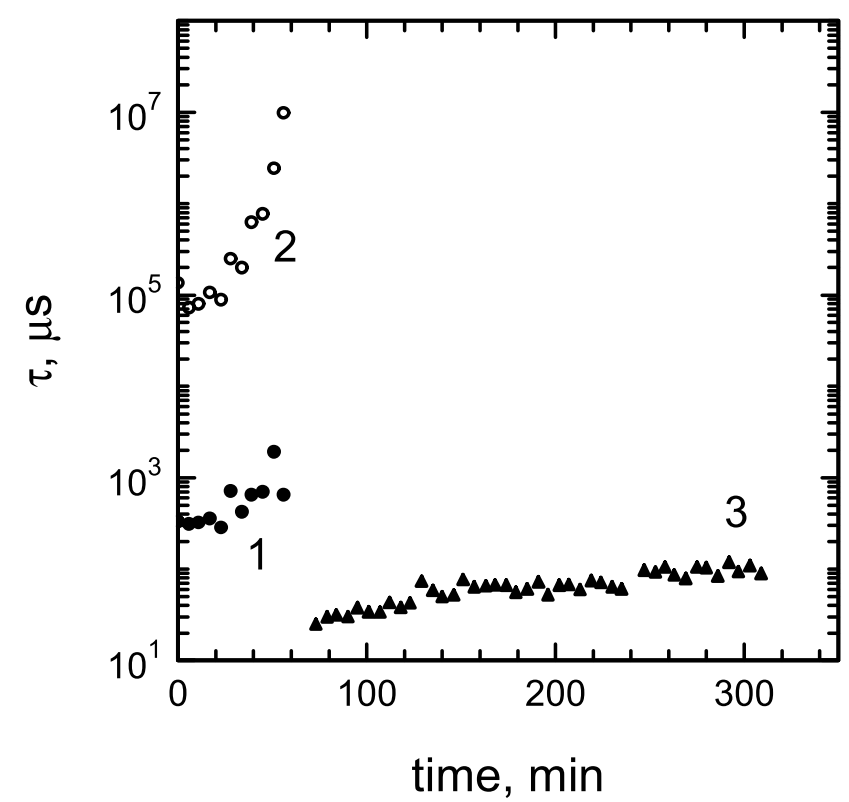

Fig. 7. Evolution of relaxation times of the three dynamic processes identified in Fig. 6, during the reaction of PAMS-DGEBA (10\% of THF).

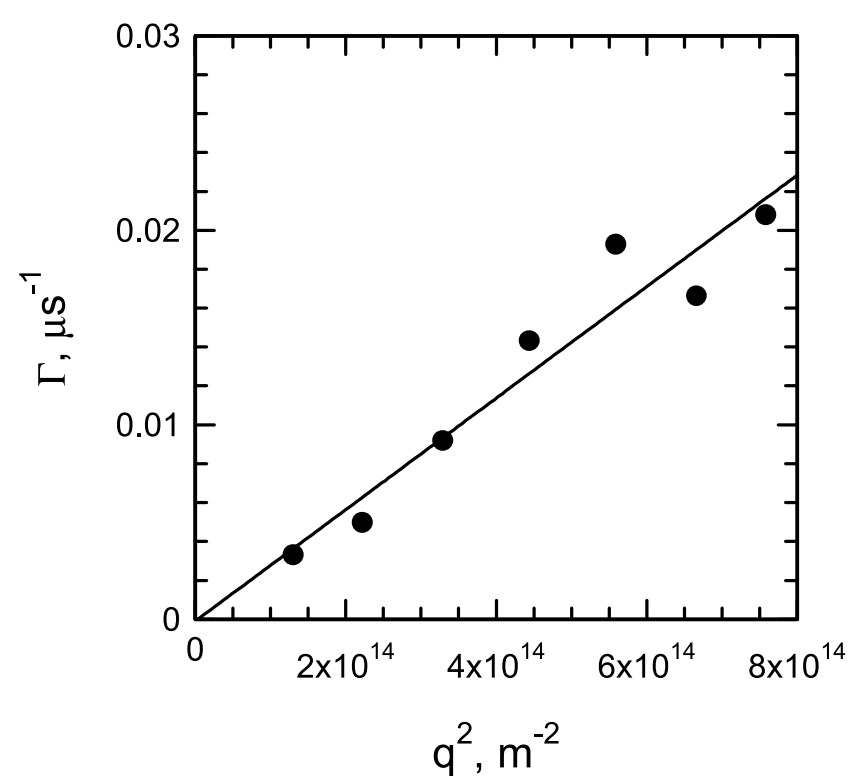

Fig. 8. Relaxation rate $\Gamma$ as a function of scattering vector in the reaction of DGEBA-PAMS (10\% of THF).

by

$$
\begin{aligned}
g^{2}(t)-1= & {\left[\beta \int \left(B \exp \left(-t / \tau_{\mathrm{f}}\right)\right.\right.} \\
& \left.\left.+(1-B)\left(1+\left(-t / \tau^{*}\right)^{\chi}\right)\right) \mathrm{d} \tau\right]^{2}
\end{aligned}
$$

where $\tau_{\mathrm{f}}$ and $\tau^{*}$ are the characteristic decay time of the fast mode and the lower cutoff of the power law behaviour.

Fitting this equation to the experimental data requires adjustment of five independent parameters (plus a possible baseline term), which does not produce very stable results given the non-negligible noise in the data. However, the noise in the correlation function is unavoidable, since the measurement time has to be restricted to no more than $300 \mathrm{~s}$, otherwise the reaction has proceeded too much. This issue is particularly important right at the gelation threshold. Therefore, we have first subtracted from the correlation function the contribution corresponding to the fast mode with relaxation time $\tau_{\mathrm{f}}$ using the subtraction technique described in Ref. [7]. The modified correlation function now represents only the power-law relaxation behavior of which a typical example is given in Fig. 9. A power-law fit to the data in Fig. 9 yields stable results, $\chi=-0.19$. The exponent $\chi$ can be related to the dynamic critical exponent for viscoelasticity $n$ by $\chi=(n-1) / 2$. From this relation we derive $n=0.62$. This value of the critical relaxation exponent is in a reasonable agreement with the values of the critical exponent found by DMA, $n=0.73$.

\subsection{Network structure, morphology and thermomechanical properties of cured systems}

Fig. 10 shows dynamic storage shear modulus $G^{\prime}(T)$ and loss factor $\tan \delta$ of PAMS-DGEBA networks of various 


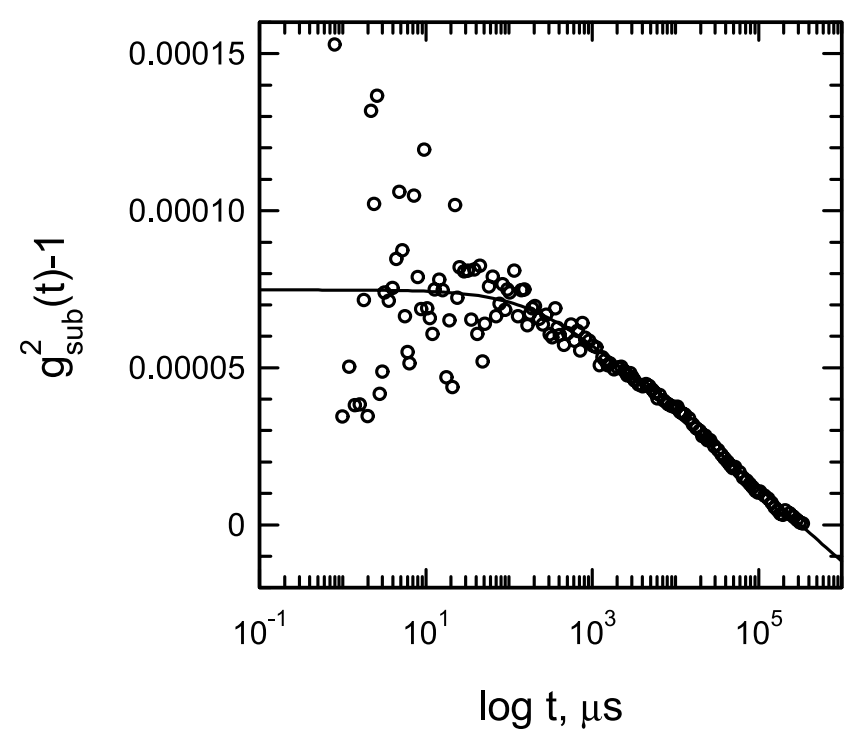

Fig. 9. Power-law behaviour of the correlation function $g_{\text {sub }}^{2}(t)$ at the gel point in the reaction of PAMS-DGEBA ( $10 \%$ of THF); for details, see the text.

compositions as a function of temperature. According to the first scan measurements, the highest modulus in the rubbery state corresponding to the highest crosslinking density was determined in the network prepared with epoxy excess, $r_{\mathrm{AE}}=0.5$ (curve 2). An increase in the crosslinking density with respect to the stoichiometric network (curve 1) is assumed to be a result of homopolymerization of the

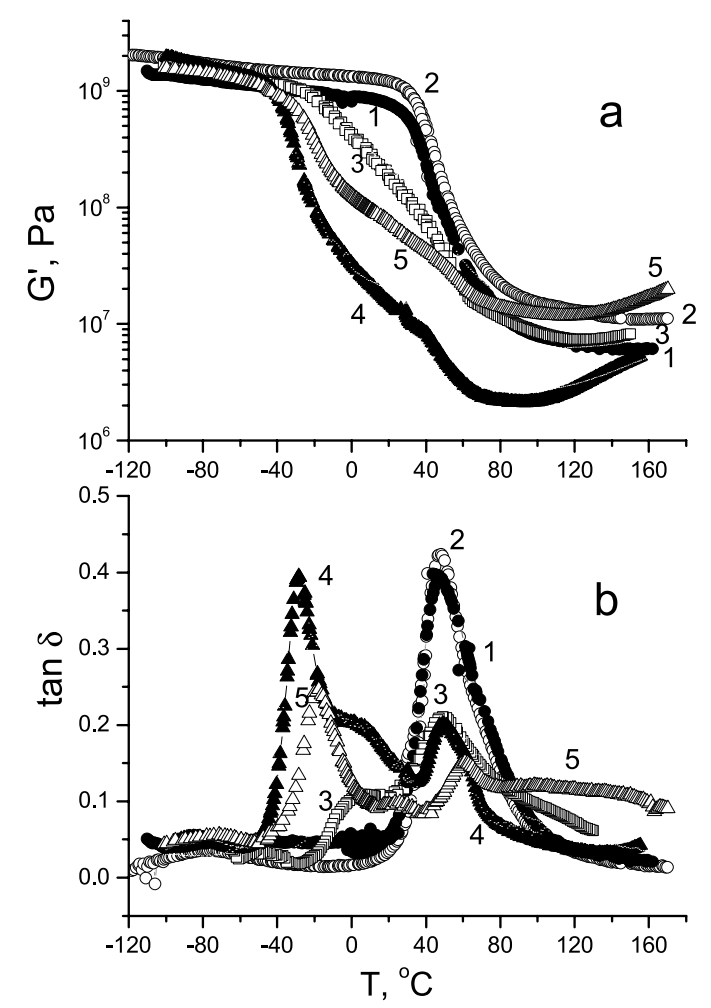

Fig. 10. Dynamic shear modulus $G^{\prime}$. (a) and loss factor $\tan \delta$, (b) of the PAMS-DGEBA networks as a function of temperature. $1, r_{\mathrm{AE}}=1 ; 2$, $r_{\mathrm{AE}}=0.5 ; 3, r_{\mathrm{AE}}=2 ; 4, r_{\mathrm{AE}}=5 ; 5, r_{\mathrm{AE}}=5$, 3rd scan. excessive epoxy groups taking place at a high curing temperature [8]. The figure shows a decrease in glass transition temperature $T_{\mathrm{g}}$ of the networks prepared with excess of amine, $r_{\mathrm{AE}}=2$ and $r_{\mathrm{AE}}=5$ (curves 3 and 4 ). Moreover, Fig. 10(b) reveals a different morphology of the PAMS-DGEBA systems as a function of composition. The stoichiometric network and that with epoxy excess exhibit one maximum of the loss factor $\tan \delta$ corresponding to a single glass transition of the network. This result seems to reveal a homogeneous phase structure in contrast to previous data [1]. However, the formed morphology depends on reaction temperature and thermal history, which were different in both cases. Moreover, it is necessary to mention that DMA does not discern two phases with a small difference in glass transition temperatures $T_{\mathrm{g}}$. The loss factor maximum at $T=50{ }^{\circ} \mathrm{C}$ is given by relaxation of the chains in the crosslinked PAMS-DGEBA phase. On the contrary, the networks with amine excess are strongly phase-separated. A more heterogeneous PAMS distribution in the off-stoichiometric networks was reported also by Cabanelas et al. [1]. Slight amine excess in the network with composition $r_{\mathrm{AE}}=2$ (curve 3 ) leads to formation of a twophase morphology with glass transitions at $T=50{ }^{\circ} \mathrm{C}$ and $T=5{ }^{\circ} \mathrm{C}$. The latter maximum could be assigned to relaxation of only partially reacted PAMS involving mainly branched but not crosslinked sequences. At a high amine excess $\left(r_{\mathrm{AE}}=5\right)$, even three relaxation maxima are observed in Fig. 10(b) (curve 4) with an additional maximum at $T=-30{ }^{\circ} \mathrm{C}$. This maximum is attributed to the glass transition of the segregated phase, which is rich in the excessive unreacted PAMS. The high amplitude of $\tan \delta$ reveals that the largest amount of PAMS is involved just in this phase. In agreement is the low modulus in the rubbery region proving low crosslinking density of this offstoichiometric PAMS-DGEBA network. The rubbery modulus, however, strongly grows with increasing temperature. This is caused by changes in the system occurring during DMA measurement carried out up to a high temperature, $170{ }^{\circ} \mathrm{C}$. The observed modulus increase cannot be explained merely by the temperature dependence of the modulus predicted in the theory of rubber elasticity; $G \sim \nu R T$, where $\nu$ is crosslinking density. The second and third measurement scans made immediately after the first one show a significantly higher rubbery modulus (see Fig. 10, curve 5). At the same time, a decrease was detected in the amplitude of $\tan \delta$ maximum both in the PAMS-rich segregated phase and mainly in the phase of partially reacted PAMS. Moreover, $T_{\mathrm{g}}$ of the crosslinked phase slightly grows to $65^{\circ} \mathrm{C}$ and a broad band spanning from 100 to $150{ }^{\circ} \mathrm{C}$ is observed. The change in thermomechanical properties, however, is partially reversible. After a sufficiently long time at room temperature, the rubbery modulus again decreases. We assume that the results could be interpreted as follows. A high temperature during DMA measurement induces postcuring and results in an increase in crosslinking density, modulus and $T_{\mathrm{g}}$, whereas the phase 
of partially reacted PAMS diminishes. In addition, the partial reversibility of the effect indicates that physical interactions play a role. The high temperature promotes a better compatibility of the separated phases and epoxy-rich and reacted PAMS-rich phases interpenetrate. The entanglements formed between the crosslinked and partially reacted PAMS phases in a state of higher miscibility may result in the temporary modulus growth.

All cured PAMS-DGEBA networks, however, show low $T_{\mathrm{g}}$ compared with the results previously published by Cabanelas et al. [1]. This glass transition decrease may be brought about by insufficient phase segregation in the studied networks. The system is quite incompatible at room temperature. However, under the curing regime used at a high temperature $\left(T=120^{\circ} \mathrm{C}\right)$ from the beginning of the cure and taking into account the grafting reaction, the blend becomes more miscible before gelation. As a result, a pronounced mixing of phases occurs mainly in the stoichiometric system leading to the network with a single glass transition. On the contrary, low initial curing temperature used by Cabanelas et al. [1] leads to a more phase separated system with a higher $T_{\mathrm{g}}$ of the epoxy-rich phase.

Crosslinked systems are usually characterized by fraction of the sol, $w_{\mathrm{S}}$, indicating defects in the network structure. In the case of the alternating amine-epoxy addition reaction, the ideal network is formed under stoichiometric composition, $r_{\mathrm{AE}}=1$. At an excess of one component in the off-stoichiometric composition the defective network is created, the sol fraction appears and at the critical ratio, $\left(r_{\mathrm{AE}}\right)_{\mathrm{C}}$, the gel is not formed at all. The theoretical dependence of the gel fraction $w_{\mathrm{g}}\left(=1-w_{\mathrm{S}}\right)$ on the PAMS-DGEBA network composition is given in Fig. 11 (curve 1). The critical ratio reflects the reaction mechanism of the network formation governing the final network structure in the same way as the critical conversion. In the case of the alternating reaction, the following relation is

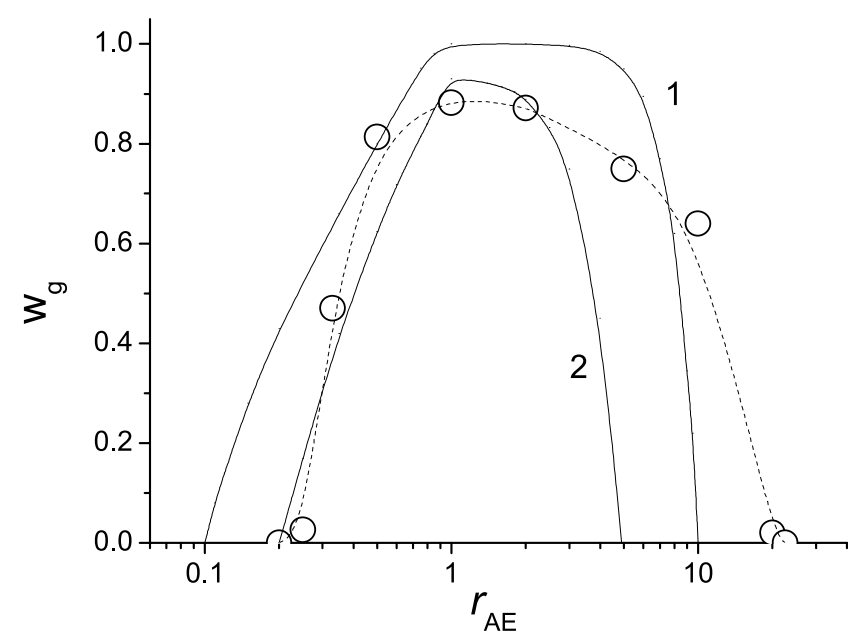

Fig. 11. Gel fraction $w_{\mathrm{g}}$ as a function of composition of the PAMS-DGEBA system. 1-theory, random reaction, 2-theory taking into account $30 \%$ of cyclization reactions. valid; $r_{\mathrm{AE}}(=\mathrm{NH} /$ epoxy $)=\alpha_{\mathrm{E}} / \alpha_{\mathrm{A}}$. Moreover, the full conversion of the minority component at the gel point is assumed [9]. Consequently, using the Eq. (2), the critical ratios could be calculated: $\left(r_{\mathrm{AE}}\right)_{\mathrm{C}}=\left(f_{\mathrm{wA}}-1\right)$ for amine excess (where $\left.\alpha_{\mathrm{E}}=1\right)$ and $\left(r_{\mathrm{AE}}\right)_{\mathrm{C}}=\left(f_{\mathrm{wA}}-1\right)^{-1}$ for epoxy excess, which corresponds to the theoretical values 10 and 0.1 , taking into account the PAMS functionality $f_{\mathrm{wA}}=11$.

For determination of the gel fraction $w_{\mathrm{g}}$, the PAMSDGEBA networks were extracted with THF and the results are given in Fig. 11. The critical ratio was evaluated by extrapolation to $w_{\mathrm{g}}=0$. In contrast to the kinetics study, these experiments were performed with bulk heterogeneous blends. Therefore, the data are affected by the phase state of the system and could provide indirect information on morphology. In the blends with amine excess, the gel is formed even at a composition with a higher than the theoretical critical ratio for the random reaction, $\left(r_{\mathrm{AE}}\right)_{\mathrm{C}}=$ 20-25 (cf. theoretical curve 1). In this case, the morphology plays an important role. In the heterogeneous blend, the reaction improves miscibility and a partially reacted PAMS becomes compatible with the minority epoxy monomer phase in contrast to unreacted PAMS. The reaction does not proceed statistically in the whole system but, due to better compatibility, DGEBA preferably reacts with the partially reacted PAMS. As a result, the initially continuous PAMS phase disintegrates during crosslinking and the incompatible domains rich in an unreacted PAMS are segregated. This separated phase was confirmed by DMA (see Fig. 10). In the epoxy-rich phase, the reaction proceeds under a lower ratio of amino and epoxy groups due to excluding the amino groups in the segregated PAMS domains. Therefore, the system gels even at a higher total value $r_{\mathrm{AE}}$ compared with the theory of the random reaction in the homogeneous system. At very high amine excess, the epoxy-rich crosslinked domains are dispersed in the PAMS matrix leading to microgel formation. The possible higher functionality of PAMS could serve as another explanation of the higher experimental value $\left(r_{\mathrm{AE}}\right)_{\mathrm{C}}$. On the contrary, in the networks with epoxy excess $\left(r_{\mathrm{AE}}<1\right)$, the gel fraction is lower compared to the theory and also the critical ratio $\left(r_{\mathrm{AE}}\right)_{\mathrm{C}}=0.24$ reveals a delay in gelation (shift closer to the stoichiometric value compared to the theoretical value 0.1 ). In this case, PAMS-rich domains are dispersed in the epoxyrich matrix [1] and the epoxy-amine reaction proceeds at the interface thus gradually compatibilizing the blend. Full conversion of the minority amino groups results in a significant homogenization of the system as proved by single glass transition in Fig. 10. We assume that the delay in gelation and a high sol fraction even in the stoichiometric network are mainly a result of the cyclization effect. Excluding the systems with high amine excess, the experimental results are in a reasonable agreement with the theoretical prediction calculated under the assumption that $30 \%$ of bonds are intramolecular (Fig. 11, curve 2). For the calculation of $w_{\mathrm{S}}$, it is equivalent to the low total conversion $(\alpha=0.7)$; however, experimentally, full 
conversion of minority groups was determined. The high sol fraction in the stoichiometric network $\left(w_{\mathrm{S}}=0.12\right)$ causes a decrease in the glass transition temperature. A possible higher functionality of PAMS does not affect data interpretation. Extent of cyclization of $30 \%$ represents the best fit even under assumption of $f_{\mathrm{wA}}=20$.

\section{Conclusions}

The epoxy-polysiloxane network was prepared by the reactive blending of the initially incompatible PAMSDGEBA system. Grafting of the polysiloxane containing pendant amino groups by the bifunctional epoxy reagent results in blend compatibilization during the reaction and in crosslinking.

The epoxy-amine reaction proceeds without any steric restrictions on the polymer chain in the pre-gel stage; however, the reaction is slowed down in the post-gel stage due to diffusion control leading possibly to concentration gradients [1]. Gelation of the system occurs at a high conversion $\left(\alpha_{\mathrm{E}}\right)_{\mathrm{C}}=0.47$ compared with the ideal random reaction $\left(\left(\alpha_{\mathrm{E}}\right)_{\mathrm{C} \text {,random }}=0.32\right)$. This delay in gelation reveals a high extent of cyclization. As a result, even the stoichiometric network contains a large fraction of the sol, $w_{\mathrm{s}}=0.12$. A reasonable agreement was obtained in predicting the gel fraction by assuming a 30\% extent of intramolecular reactions. The relatively high gel fraction in the networks with PAMS excess is explained by segregation of the PAMS-rich phase thus decreasing the effective NH/ epoxy ratio in the miscible reactive domain.

The structure and morphology evolution during network formation in the blend bulk and in a partly homogenized THF solution (10 or $20 \%$ of THF) are governed by several competing processes. In the bulk, compatibilization of an initially heterogeneous blend takes place during crosslinking. In the solution, polymer structure growth and crosslinking tend to phase separation from solution and the epoxy-amine grafting reaction results in compatibilization of the reactive blend. Chemorheology and light scattering results proved early phase separation of the growing PAMSDGEBA clusters followed by subsequent compatibilization and gelation. The fast and slow relaxation modes corresponding to diffusion of the unreacted reagents and PAMSDGEBA clusters, respectively, were determined by DLS in the pregel-stage. The clusters in THF solution grow from the initial size $R \sim 100 \mathrm{~nm}$. Only a single relaxation mode was observed in the post-gel stage assigned to the chains between the network crosslinks Light scattering techniques TRLS and DLS were shown to provide a sensitive information on the gel point. The sudden increase in intensity of the scattered light, increasing width of the distribution of relaxation times of species in the system resulting in appearance of the power-law behavior, and a sharp decrease in the amplitude of the correlation function (parameter $\beta$ in Eq. (1)) determined in DLS are the phenomena characterizing the point of gelation.

Morphology and properties of the cured PAMS-DGEBA blend depend on composition. The networks with stoichiometric composition exhibit the most homogeneous morphology with a single glass transition temperature. Excess of amine (PAMS) results in a strongly phase-separated system. Both unreacted PAMS-rich domains and the phase of partially grafted PAMS are segregated. Heating of the cured system to a high temperature leads to a reversible phase interpenetration, entanglements formation and an increase in modulus.

\section{Acknowledgements}

The authors acknowledge financial support of the European Commission through a Marie Curie Training Site contract No. HPMT-CT-2001-00396 and of the Grant Agency of Academy of Sciences of the Czech Republic (A4050008).

\section{References}

[1] Cabanelas JC, Serrano B, González-Benito J, Bravo J, Baselga J. Macromol Rapid Commun 2001;22:694

[2] Kichler A, Sabourault N, Décor R, Leborgne Ch, Schmutz M, Valleix A, Danos O, Wagner A, Mioskowski Ch. J Controlled Release 2003;93: 403.

[3] Jakeš J. Czech J Phys 1988;B38:1305.

[4] Malkin AYa, Kulichikhin SG. In: Karlsruhe DE, editor. Rheokinetics. Huthig and Wepf Verlag; 1996.

[5] Winter HH, Mours M. Adv Polym Sci 1997;134:165.

[6] Stockmayer WH. J Polym Sci 1952;9:69.

[7] Štěpánek P, Johnsen R. Collect Czech Chem Commun 1995;60:1941.

[8] Matějka L. Macromolecules 2000;33:3611.

[9] Matějka L, Dušek K. Polym Bull 1980;3:489. 